\title{
Zebrafish modeling reveals that SPINT1 regulates the aggressiveness of skin cutaneous melanoma and its crosstalk with tumor immune microenvironment
}

\author{
Elena Gómez-Abenza ${ }^{1,2}$, Sofía Ibáñez-Molero 1,2, Diana García-Moreno 1,2, Inmaculada Fuentes ${ }^{1,2}$, Leonard I. Zon 3,4,
} Maria C. Mione ${ }^{5}$, María L. Cayuela ${ }^{6}$, Chiara Gabellini ${ }^{1,2,7^{*}}$ and Victoriano Mulero ${ }^{1,2^{*}}$ (i)

\begin{abstract}
Background: Skin cutaneous melanoma (SKCM) is the most lethal form of skin cancer and while incidence rates are declining for most cancers, they have been steadily rising for SKCM. Serine protease inhibitor, kunitz-type, 1 (SPINT1) is a type II transmembrane serine protease inhibitor that has been shown to be involved in the development of several types of cancer, such as squamous cell carcinoma and colorectal cancer.

Methods: We used the unique advantages of the zebrafish to model the impact of Spintla deficiency in early transformation, progression and metastatic invasion of SKCM together with in silico analysis of the occurrence and relevance of SPINT1 genetic alterations of the SKCM TCGA cohort.

Results: We report here a high prevalence of SPINT1 genetic alterations in SKCM patients and their association with altered tumor immune microenvironment and poor patient survival. The zebrafish model reveals that Spint1a deficiency facilitates oncogenic transformation, regulates the tumor immune microenvironment crosstalk, accelerates the onset of SKCM and promotes metastatic invasion. Notably, Spint1a deficiency is required at both cell autonomous and non-autonomous levels to enhance invasiveness of SKCM.
\end{abstract}

Conclusions: These results reveal a novel therapeutic target for SKCM.

Keywords: SPINT1, Melanoma, Inflammation, Tumor stroma, Epithelial to mesenchymal transition, Metastasis, Macrophages, Zebrafish

\section{Background}

Skin cutaneous melanoma (SKCM) originates from melanocytes, neural-crest derived pigment-producing cells located in the epidermis, where their major function is to protect keratinocytes from UV-induced DNA damage [1]. The malignant transformation of melanocytes generates this fatal form of skin cancer with a complex multigenic etiology that becomes extremely difficult to treat once it has metastasized. SKCM is the deadliest form of skin cancer $(75 \%$ of deaths related to skin cancer) and it is common in the Western world.

\footnotetext{
*Correspondence: chiara.gabellini@unipi.it; vmulero@um.es 'Departamento de Biología Celular e Histología, Facultad de Biología, Universidad de Murcia, Murcia, Spain

Full list of author information is available at the end of the article
}

Indeed, its global incidence is $15-25$ per 100,000 individuals [2]. While incidence rates are declining for most cancers, they have been steadily rising for SKCM worldwide [3]. Early detection is fundamental, since localized, early stage SKCM can be surgically excised with little chance of recurrence with a $98.2 \%$ of patient survival rate after 5-year survival as reported by The Surveillance, Epidemiology, and End Results (SEER) [4]. Metastatic SKCM, however, is still an often fatal disease with a 5-year survival rate of $15-20 \%$ [3].

SKCM is one of the most recurrent types of cancer and its genetic heterogeneity has led in recent years to join forces to determine SKCM causes and develop effective therapies. Transformation of melanocytes into primary and then metastatic SKCM requires a complex

(c) The Author(s). 2019 Open Access This article is distributed under the terms of the Creative Commons Attribution 4.0 International License (http://creativecommons.org/licenses/by/4.0/), which permits unrestricted use, distribution, and 
interplay of exogenous and endogenous events [5]. More than $50 \%$ of the tumors originate from normal skin rather than from dysplastic nevi, suggesting that SKCM not only appears to be due to the transformation of mature melanocytes [6]. In this way, the identification of SKCM initiating cells is really important to devising methods for early detection and eradication of SKCM [7, 8]. Moreover, SKCM stem cell populations have been characterized and associated with tumor progression, immunoevasion, drug resistance and metastasis [9].

Inflammation can play a key role in cancer, from initiation of the transformed phenotype to metastatic spread. Nevertheless, inflammation and cancer have a profound yet ambiguous relationship. Inflammation (especially chronic inflammation) has protumorigenic effects, but inflammatory cells also mediate an immune response against the tumor and immunosuppression is known to increase the risk for certain tumors [10]. Nowadays, skin cancers are also attributed to chronically injured or nonhealing wounds and scars or ulcers that occur at sites of previous burns, sinuses, trauma, osteomyelitis, prolonged heat and chronic friction. The incidence of malignancy in scar tissues is $0.1-2.5 \%$ and it is estimated that underlying infections and inflammatory responses are linked to $15-20 \%$ of all deaths from cancer worldwide [11]. Furthermore, chronic inflammation contributes to about $20 \%$ of all human cancers [12].

Serine protease inhibitor, kunitz-type, 1 (SPINT1), also known as hepatocyte growth factor activator inhibitor 1 (HAI1), is a type II transmembrane serine protease inhibitor that plays a crucial role in the regulation of the proteolytic activity of both suppression of tumorigenicity 14 (ST14), also known as Matriptase-1 [13-15], and Hepatocyte growth factor activator (HGFA) [16]. The functional linkage between ST14 and SPINT1 has important implications for the development of cancer. ST14 activity, which is only partially opposed by endogenous SPINT1, causes increased sensitivity to carcinogens and produces spontaneous tumorigenesis in the skin of keratin-5-matriptase transgenic mice, while increased epidermal SPINT1 expression fully counteracts the oncogenic effect of ST14 [17]. Furthermore, the expression of ST14 has been demonstrated to be up-regulated in various human cancer histotypes such as breast, cervix, ovaries, prostate, esophagus and liver cancers [18].

The close functional relationship between ST14 and SPINT1 was also observed in a zebrafish model of skin inflammation, carrying a hypomorphic mutation of spint1a. Indeed epidermal hyperproliferation and neutrophil infiltration observed in mutant zebrafish larvae are both rescued by $s t 14 a$ gene knock-down, suggesting a novel role for the SPINT1-ST14 axis in regulating inflammation $[19,20]$. Given the unique advantages of the zebrafish model for tumor cell transplantation, thanks to the transparency of embryos and even adults that allows the tracking of donor cells and the strong correlation between alterations of Spint1a-St14a levels with tumor progression, the spint1a mutant zebrafish represents an attractive model to study the role of SPINT1 and chronic inflammation in SKCM.

Our results show that genetic alterations of SPINT1 correlated with a poor prognosis of SKCM patients and provide evidence that SPINT1 expression positively correlated with tumor macrophage infiltration, but not neutrophils. In line with these clinical data, we show that Spintla deficiency enhances at both cell autonomous and non-autonomous levels cell dissemination of SKCM in zebrafish models by promoting tumor dedifferentiation and altered immune surveillance.

\section{Methods \\ Animals}

Wild-type zebrafish (Danio rerio H. Cypriniformes, Cyprinidae) were obtained from the Zebrafish International Resource Center (ZIRC, Oregon, USA) and mated, staged, raised and processed as described in the zebrafish handbook [21]. Zebrafish fertilized eggs were obtained from natural spawning of wild type and transgenic fish held at our facilities following standard husbandry practices. Animals were maintained in a $12 \mathrm{~h}$ light/dark cycle at $28^{\circ} \mathrm{C}$. Tg(kita:GalTA4,UAS:mCherry $)^{\text {hzm1 }}$ zebrafish were crossed with $T g\left(U A S: e G F P-H-R A S \_G 12 V\right)^{i o 6}$ line [8] to express oncogenic human HRAS_G12 V driven by the melanocyte cell-specific promoter kita. Transgene expression was confirmed in 3 day post-fertilization larvae (dpf) by fluorescence microscopy and by analyzing melanocyte hyperproliferation [8]. The hi2217 line, which carries a hypomorphic spint1a mutant allele that promotes skin inflammation [19], and transparent roy ${ }^{a 9 / a 9} ; n a c r e^{w 2 / w 2}$ (casper) [22] of 4-8 month old were previously described.

Zebrafish larvae were anesthetized by a solution of $0.16 \mathrm{mg} / \mathrm{ml}$ buffered tricaine (Sigma-Aldrich) in embryo medium. Adult zebrafish were anesthetized by a dual anesthetic protocol to minimize over-exposure to tricaine, in long-term studies (up to $40 \mathrm{~min}$ ) [23]. Briefly, the anesthesia was firstly induced by tricaine and then fish were transferred to tricaine/isoflurane solution (forane in ethanol, 1:9).

\section{Tumor sampling, disaggregation and cell sorting}

Primary melanoma tumors were excised from adult zebrafish once they had reached between 3 and $5 \mathrm{~mm}$ in diameter. Some individuals were euthanized according the European Union Council and IUAC protocol and others were monitored and maintained still alive after the tumor biopsy treated with conditioners to reduce fish stress and heal damaged tissue and wounds (STRESS COAT, API), as well as to protect from bacterial (MELAFIX, API) and fungal infections (PIMAFIX, API). 
The tumor was excised with a clean scalpel and razor blade, placed in $2 \mathrm{ml}$ of dissection media, composed by DMEM/F12 (Life Technologies), $100 \mathrm{UI} / \mathrm{ml}$ penicillin, $100 \mu \mathrm{g} / \mathrm{ml}$ streptomycin, $0.075 \mathrm{mg} / \mathrm{ml}$ Liberase (Roche). After manually disaggregation with a clean razor blade and incubation at room temperature for $30 \mathrm{~min}, 5 \mathrm{ml}$ of wash media, composed by DMEM/F12 (Life Technologies), penicillin-streptomycin (Life Technologies), and 15\% heat-inactivated FBS (Life Technologies), were added to the tumor slurry and manually disaggregated one last time. Next, the tumor cell suspensions were passed through a $40 \mu \mathrm{m}$ filter (BD) into a clean $50 \mathrm{ml}$ tube. An additional $5 \mathrm{ml}$ of wash media was added to the initial tumor slurry and additionally filtered. This procedure was repeated twice. Cells were counted with a hemocytometer and the tubes of resuspended cells were centrifuged at $800 \mathrm{~g}$ for $5 \mathrm{~min}$ at $4{ }^{\circ} \mathrm{C}$. The pellets of tumor cells were resuspended in PBS containing 5\% FBS and kept on ice prior to transplantation [23].

The resulting cell suspension from zebrafish melanoma tumors was passed through a $40 \mu \mathrm{m}$ cell strainer and propidium iodide (PI) was used as a vital dye to exclude dead cells. The Cell Sorting was performed on a "Cell Sorter" SONY SH800Z in which eGFP positive cells were sorted from the negative ones of the same cell tumor suspension.

\section{Larval allotransplantion assays}

Melanomas were disaggregated, then labelled with $1,1^{\prime}$ di-octa-decyl-3,3,3', 3' -tetra-methyl-indo-carbo-cya-nine perchlorate (DiI, ThermoFisher) and finally resuspended in a buffer containing 5\% FBS in PBS. Between 25 to 50 cells/embryo were then injected in the yolk sac of 60-90 Casper or spint1 mutants zebrafish larvae of $48 \mathrm{~h}$ postfertilization (hpf) and after 5 days at $28^{\circ} \mathrm{C}$, larvae were scored manually in blind samples for zebrafish melanoma cells dissemination by fluorescence microscopy [24]. Melanoma cell invasion score was calculated as the percentage of zebrafish melanoma cell-invaded larvae over the total number of larvae analyzed taking into account also the number of tumor foci per larvae. Three tumor foci were established to score a larva as positive for invasion. Furthermore, larvae positive for invasion were also distinguished in three groups considering the number of positive foci per larvae: $3-5$ foci per larvae, 5-15 foci per larvae and > 15 foci per larvae.

\section{Adult allotransplantion assays}

Adult zebrafish used as transplant recipients were immunosuppressed to prevent rejection of the donor material. Thus, the recipients were anesthetized, using the dual anesthetic protocol described above, and treated with 30 Gy of split dose sub-lethal X-irradiation (YXLON SMART $200 \mathrm{E}, 200 \mathrm{kV}, 4.5 \mathrm{~mA}) 2$ days before the transplantation.
Then the immunosuppressed fish were maintained in fresh fish water treated with conditioners preventing infections onset and the consequent recipient deaths.

Anesthetized fish (10-20 per tumor) were placed dorsal side up on a damp sponge and stabilized with one hand. Using the other hand, the needle of a $10 \mu \mathrm{l} \mathrm{bev-}$ eled, 26S-guaged syringe (Hamilton) was positioned midline and ahead to the dorsal fin. 30,000, 100,000, 300,000 and 500,000 cells resuspended in PBS were injected into the dorsal subcutaneous cavity. The syringe was washed in $70 \%$ ethanol and rinsed with PBS between uses. Following transplantation, fish were placed into a recovery tank of fresh fish water and kept off-flow with daily water changes for 7 days. Large and pigmented tumors engrafted and were observed to expand by 10 days post-transplantation.

\section{SKCM imaging in adult zebrafish}

Adult zebrafish were scored weekly for melanoma formation starting at the first appearance of raised lesions. Tumor scoring was blinded and experiments were independently repeated at least 3 times. Zebrafish were anesthetized, placed in a dish of fish water and photographed using a mounted camera (Nikon D3100 with a Nikon AF-S Micro Lens). The pigmented tumor size was represented as the number of pigmented pixels (Adobe Photoshop CS5).

\section{Analysis of gene expression}

Once zebrafish tumors reached between 3 and $5 \mathrm{~mm}$ of diameter, they were excised and total RNA was extracted with TRIzol reagent (Invitrogen), following the manufacturer's instructions, and then treated with DNase I, amplification grade $(1 \mathrm{U} / \mu \mathrm{g}$ RNA; Invitrogen). SuperScript III RNase $\mathrm{H}^{-}$Reverse Transcriptase (Invitrogen) was used to synthesize first-strand cDNA with oligo $(\mathrm{dT})_{18}$ primer from $1 \mu \mathrm{g}$ of total RNA at $50{ }^{\circ} \mathrm{C}$ for $50 \mathrm{~min}$. Realtime PCR was performed with an ABI PRISM 7500 instrument (Applied Biosystems) using SYBR Green PCR Core Reagents (Applied Biosystems). Reaction mixtures were incubated for $10 \mathrm{~min}$ at $95^{\circ} \mathrm{C}$, followed by 40 cycles of $15 \mathrm{~s}$ at $95^{\circ} \mathrm{C}, 1 \mathrm{~min}$ at $60^{\circ} \mathrm{C}$, and finally $15 \mathrm{~s}$ at $95^{\circ} \mathrm{C}, 1 \mathrm{~min} 60^{\circ} \mathrm{C}$ and $15 \mathrm{~s}$ at $95^{\circ} \mathrm{C}$. For each mRNA, gene expression was normalized to the ribosomal protein S11 gene (rps11) content in each sample Pfaffl method [25]. The primers used are shown in Additional file 1: Table S1. In all cases, each PCR was performed with triplicate samples and repeated at least in two independent samples.

\section{Human SKCM dataset analysis}

Normalized gene expression, patient survival data, genetic alterations and neutrophil/macrophage infiltration were downloaded on 14th December 2017 from SKCM repository of The Cancer Genome Atlas (TCGA, 
Provisional) from cBioPortal database (https://www.cbio portal.org/). This cohort includes 479 patient samples. No cleaning filter was applied and all patient data available for each study were used: 469 samples for expression analysis, 461 samples for survival analysis and 468 for immune cell infiltration. Transcript levels of SPINT1 in human samples from normal skin, benign and malignant melanoma was collected from Gene Expression Omnibus (GDS1375 dataset and 202826_at probe). Gene expression plots and regression curves for correlation studies were obtained using GraphPad Prism 5.03 (GraphPad Software).

\section{Statistical analysis}

Data are shown as mean \pm SEM and they were analyzed by analysis of variance (ANOVA) and a Tukey multiple range test to determine differences between groups. The survival curves were analysed using the log-rank (Mantel-Cox) test. All the experiments were performed at least three times, unless otherwise indicated. The sample size for each treatment is indicated in the graph and/or in the figure legend. Statistical significance was defined as $p<0.05$.

\section{Results}

SPINT1 genetic alterations are associated with poor prognosis of SKCM patients and altered tumor immune microenvironment

To study the impact of SPINT1 in promoting SKCM progression and aggressiveness, an in silico analysis of human SKCM samples of the TCGA cohort was performed. This analysis revealed that genetic alterations occurred in 10\% of SKCM patients; a relevant percentage comparing with major SKCM driven oncogenes and tumor suppressors (Fig. 1a). Among these genetic alterations, an increased mRNA level was the most prevalent alteration (7\%), while $1.9 \%$ missense mutations of unknown significance and $1.9 \%$ deep deletions were also observed. Notably, these genetic alterations of SPINT1 significantly correlated with poor SKCM patient prognosis (Fig. 1b) and SPINT1 expression was significantly inhibited in human SKCM comparing with nevus and normal skin (Fig. 1c). In addition, the analysis of patient survival with increased SPINT1 transcript levels also revealed their poor prognosis but, unfortunately, the analysis of deep deletions and missense mutations separately gave no statistical significance because the number of patients for each condition was very low (data not shown). We next performed a GO enrichment analysis of biological process (Fig. 1d), analyzing the differentially expressed genes in SKCM samples of the TCGA cohort with missense mutations or copy-number alterations of SPINT1. The results showed that regulation of immune system, inflammatory response, cell cycle, cell adhesion, and extracellular matrix organization represent key pathways significantly affected in human SKCM with these SPINT1 genetic alterations. Collectively, these results suggest that both high and low levels of SPINT1 result in an unbalanced crosstalk between tumor cells and their microenvironment promoting higher aggressiveness.

The tumor microenvironment contains diverse leukocyte populations, including neutrophils, eosinophils, dendritic cells, macrophages, mast cells and lymphocytes [26]. It is known that tumor-associated macrophages (TAM) are able to interact with tumor cells and can promote cancer progression [27-29]. As shown in Fig. 1e, the number of TAM in human SKCM samples correlated with the mRNA levels of SPINT1 in metastatic SKCM. However, the number of tumor-associated neutrophils (TAN) was independent of SPINT1 levels in both primary and metastatic SKCM. These data further confirmed the role of SPINT1 in the regulation of the crosstalk between tumor and inflammatory cells in human SKCM.

\section{The expression of SPINT1 positively correlates with both inflammation and macrophage markers in human SKCM biopsies}

In order to further understand the role of SPINT1 in SKCM, the RNA Seq database of the large TCGA cohort of SKCM was analyzed in terms of the expression of SOX10, TYR and DCT genes, that have been shown to be important in melanocyte development [30,31]. In addition, SOX10 is a recognized biomarker for the diagnosis of SKCM [30]. It was found that SPINT1 expression positively correlated with those of SOX1O and TYR, while a negative correlation was found between the expression of SPINT1 and DCT (Fig. 2a). The expression of the epithelial to mesenchymal transition (EMT) markers ZEB1, ZEB2 and TWIST1, but not TWIST2, negatively correlated with that of SPINT1 in SKCM (Fig. 2b).

SKCM cells release several cytokines and chemokines that recruit and polarize macrophages [32]. Therefore, several inflammation markers were analyzed and only the expression of the genes encoding the receptor of the pro-inflammatory cytokine TNF $\alpha$ (TNFR1) and the receptor of the pro-inflammatory chemokine interleukin 8 (CXCR2), positively correlated with SPINT1 levels (Fig. 2c). Notably, the macrophage marker MFAP4 also positively correlated with SPINT1 expression (Fig. 2d). However, the M2 polarization marker CD163 (Fig. 2d) and several interferonstimulated genes (ISGs) (Fig. 2e) were all unaffected by SPINT1 levels. Collectively, these results further suggest that SPINT1 regulates SKCM differentiation and aggressiveness, and macrophages infiltration.

\section{Inflammation accelerates the onset of SKCM in zebrafish}

Given the strong correlation between alterations of SPINT1 levels with the progression of SKCM and the crosstalk with the tumor immune microenvironment, we crossed the 


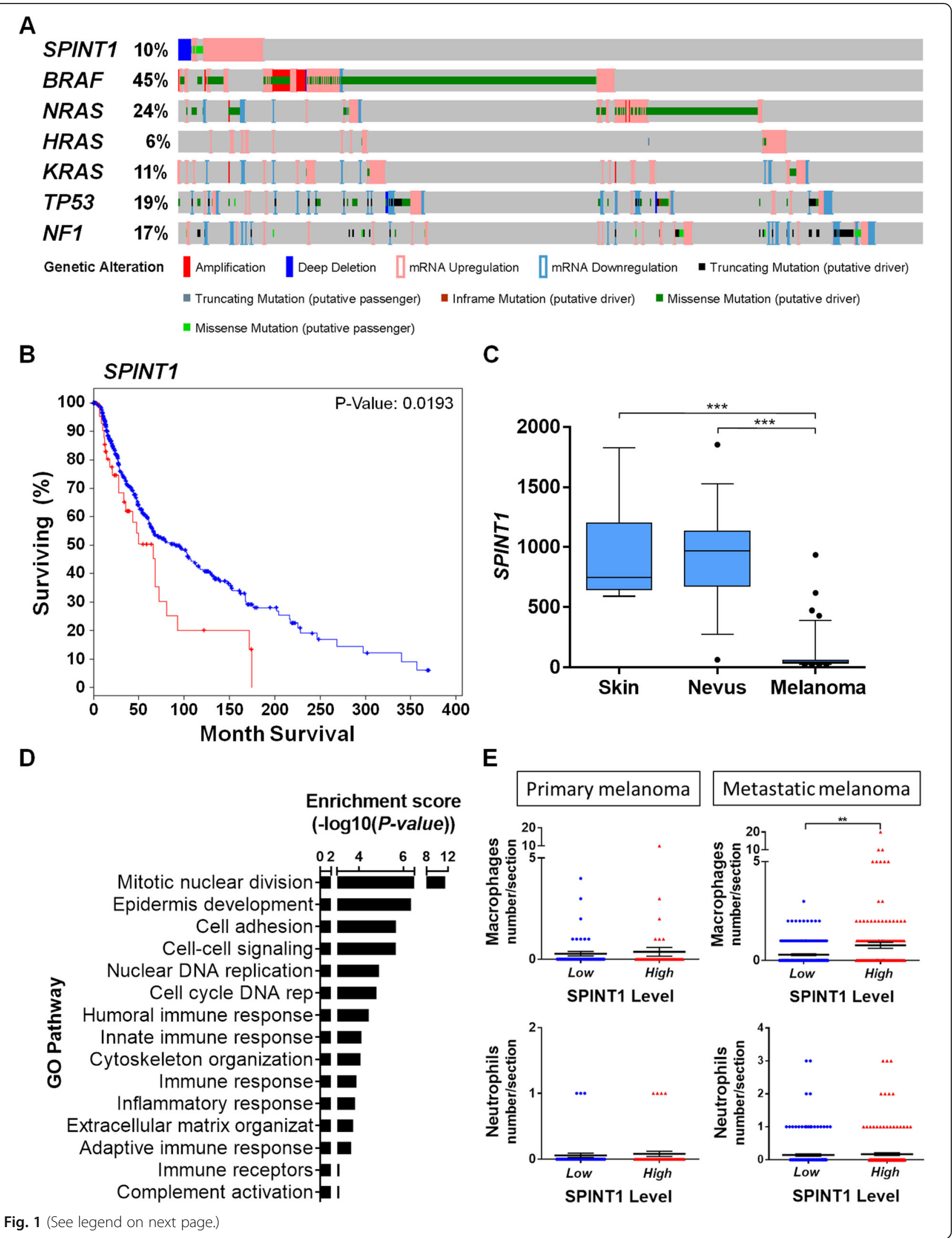


(See figure on previous page.)

Fig. 1 SPINT1 genetic alterations are associated with poor prognosis of SKCM patients. a Percentage of genetic alterations in oncogenes, tumor suppressor genes and SPINT1 in SKCM patients of the TCGA cohort $(n=479)$. $\mathbf{b}$ Survival curve of patients with genetic alteration (increased mRNA level, missense mutations and deep deletions, red line) vs. wild type (blue line) SPINT1 of SKCM of the TCGA cohort. Kaplan-Meier Gehan-BreslowWilcoxon and nonparametric Log-rank Test. Hazard ratio $=1.625 ; 95 \% \mathrm{Cl}$ of ratio $=1.099-3.033$. c Genetic expression of SPINT1 in human samples from normal skin, nevus and malignant melanoma from GEO data set GDS1375 and 202826_at probe $(n=70)$. ${ }^{* * *} p<0.001$ according to ANOVA and Tukey's Multiple Comparison Test. $\mathbf{d}$ Enrichment analysis of GO biological process. Representation of the most significant GO biological process altered when SPINT1 is affected by missense mutations or copy-number alterations. Analysis Type: PANTHER Overrepresentation Test (Released 05/12/2017), Test Type: FISHER. e Number of infiltrated macrophages and neutrophils in SKCM samples of the TCGA cohort ( $n=468)$. The number of infiltrated cells in SKCM samples with low (blue) or high (red) SPINT1 mRNA levels according to the median. The mean \pm S.E.M. for each group is shown. ${ }^{*} p<0.05 ;{ }^{*} p<0.01$ according to Student $t$ Tests

zebrafish line kita:Gal4;HRAS-G12V, which expresses the human oncogene HRAS-G12V in melanocytes and spontaneously develops SKCM [8], with the zebrafish mutant line spint1a ${ }^{\text {hi2217Tg/hi2217Tg }}$ [19], which presents chronic skin inflammation (Fig. 3a). Firstly, we quantified by fluorescence microscopy the number of early oncogenically transformed goblet cells, which also expressed the kita promoter [33], in spint1a-deficient larvae and their wild type siblings (Fig. 3b). The results showed that spint1a deficiency resulted in increased number of HRAS-G12V ${ }^{+}$cells (Fig. 3c). To determine if the enhanced Spintla deficiency-driven oncogenic transformation was also able to promote SKCM aggressiveness, SKCM development in spint1a hi2217Tg/hi2217Tg fish were compared with wild type (spint1a $a^{+ \text {hi2217Tg }}$ ) from the end of metamorphosis stage (between 28 and $30 \mathrm{dpf}$ ) to $120 \mathrm{dpf}$ (adult stage) (Fig. 3d-f). The resulting Kaplan-Meier curve showed a significant increased incidence of melanoma in the Spint1a-deficient fish, which developed SKCM in more than $50 \%$ of cases at 50-60 dpf compared with their wild type siblings which reached only $30 \%$ at this age (Fig. 3f). These data suggest that Spintla deficiency increases oncogenic transformation and accelerates SKCM onset in vivo.

\section{Spint1a deficiency is required at cell autonomous and non-autonomous levels to enhance SKCM cell dissemination in a zebrafish larval allotrasplantation model}

To assess the in vivo role of Spintla deficiency in SKCM in-

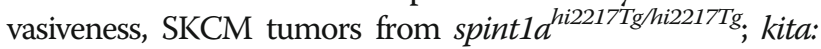

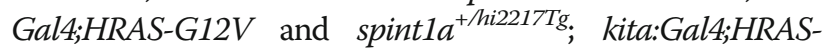
$G 12 \mathrm{~V}$ were disaggregated, after staining the cells were transplanted into the yolk sac of $2 \mathrm{dpf}$ casper zebrafish larvae (Fig. 4a). The results showed that Spintla deficiency in SKCM cells enhanced the dissemination of SKCM, assayed as the percentage of invaded larvae and the number of foci per larva, compared to control SKCM cells (Fig. 4b-d). We next examined whether Spintla deficiency in the stroma, i.e. in a non-autonomous manner, also promoted SKCM aggressiveness. Spintla wild type SKCMs were transplanted into the yolk sac of Spintla-deficient and their wild type siblings larvae (Fig. 4e). Strikingly, it was found that Spint1a deficiency in the tumor microenvironment also promoted a significantly higher dissemination of SKCM compared to control tumor microenvironments (Fig. 4f-h).

To further confirm a role of Spint1a in both SKCM and tumor microenvironment cells, we next sorted tumor $\left(\mathrm{eGFP}^{+}\right)$and stromal $\left(\mathrm{eGFP}^{-}\right)$cells from both genotypes and then mixed in equal proportions $(\sim 90 \%$ of tumor and $\sim 10 \%$ of stromal cells) in the 4 possible combinations (Fig. 5a), since it was found that all tumors had $\sim 90 \%$ of tumor and $\sim 10 \%$ of stromal cells (data not shown). Notably, both Spintla-deficient tumor and stromal cells were able to increase SKCM cell invasion (Fig. 5b and c). Collectively, these results suggest that Spintla deficiency enhances SKCM invasion by both cell autonomous and non-autonomous mechanisms.

\section{Spint1a-deficient SKCM cells showed enhanced aggressiveness in adult zebrafish allotransplantation model}

The results obtained in allotransplantation assay in larvae prompted us to analyze the role of Spint1a in SKCM aggressiveness and metastasis in adult casper zebrafish to directly visualize tumor cell proliferation and dissemination over time. spint $1 a^{\text {hi2217Tg/hi2217Tg }}$ and spint $1 a^{+/-}$ hi2217Tg SKCMs were sampled, disaggregated and subcutaneously injected $(300,000$ cells $)$ in the dorsal sinus of adult casper recipients previously irradiated with 30 Gy (Fig. 6a). Tumor engraftment was visible as early as 7 days post-transplantation in both genotypes. While $90 \%$ engraftment was obtained with wild type SKCM cells, Spintla-deficient cells showed a significant enhancement of tumor engraftment rate, around 95\% (Fig. 6b). In addition, adult zebrafish recipients transplanted with Spint1a-deficient SKCMs developed tumors with a significant higher growth rate than those injected with wild type SKCMs (Fig. 6c). Notably, Spintla-deficient SKCM cells were able to invade the entire dorsal area, part of ventral cavity and the dorsal fin (Fig. 6c).

We next performed additional transplant assays following the same work-flow but injecting an increased number of cells (500,000 cells per recipient fish), that ensured a $100 \%$ of engraftment was for both genotypes (data not shown). From the first week of analysis, Spint1a-deficient SKCM tumor size was significantly 
A

Melanocyte differentiation markers
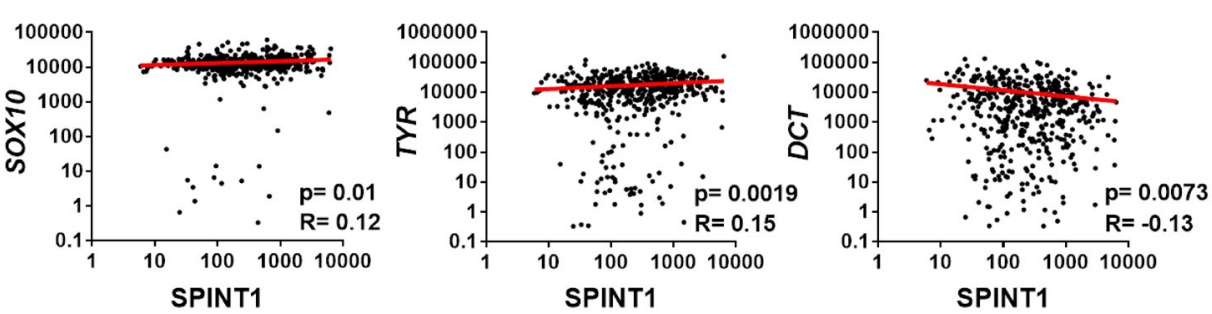

B
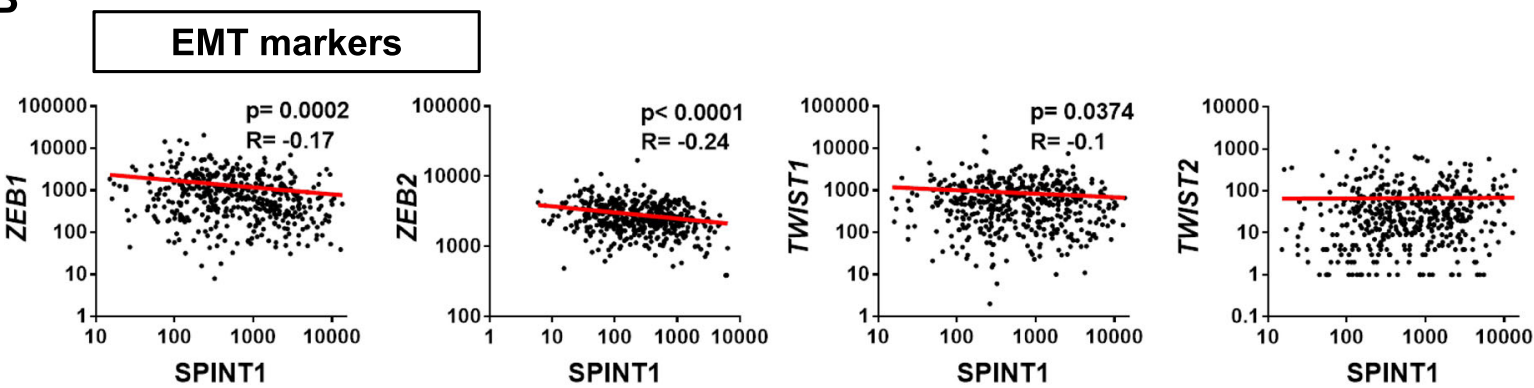

C
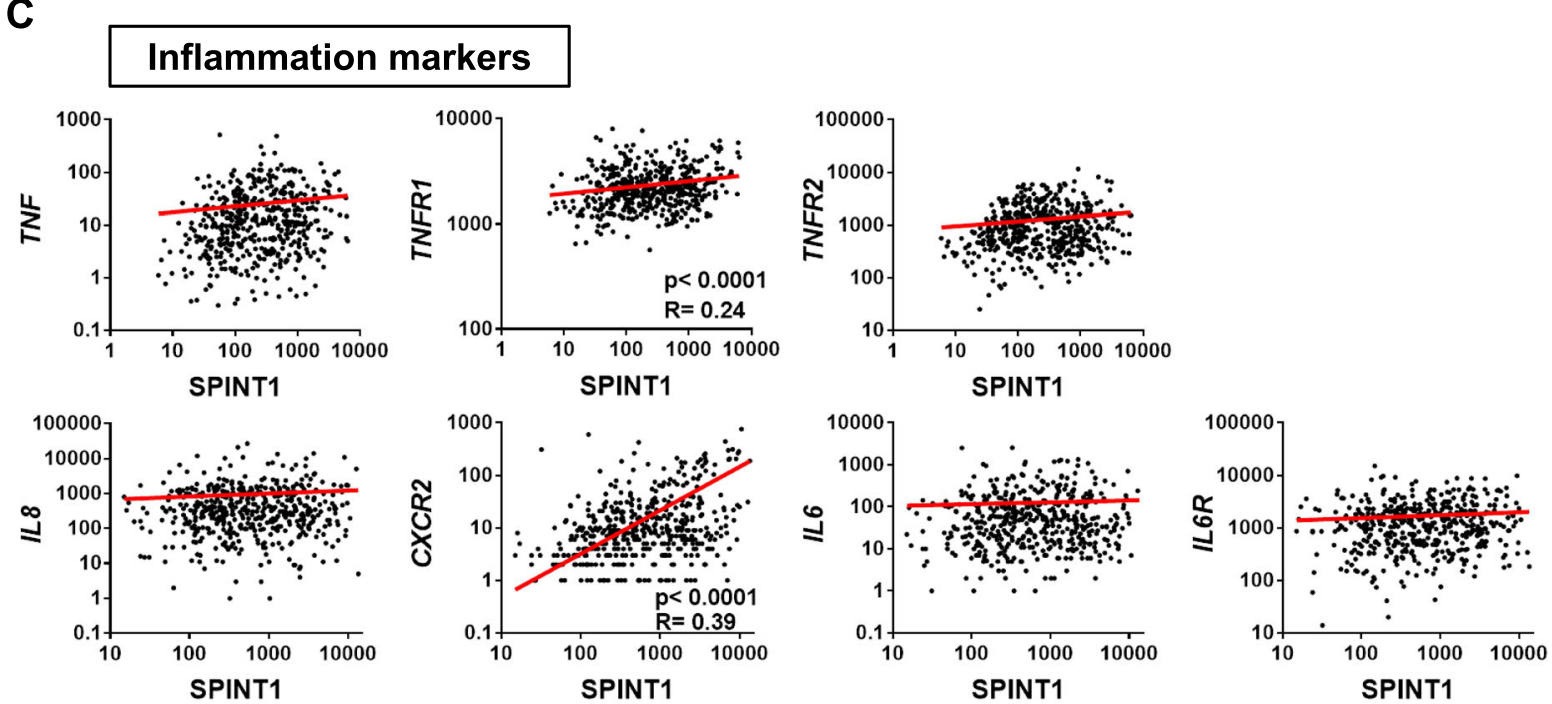

D
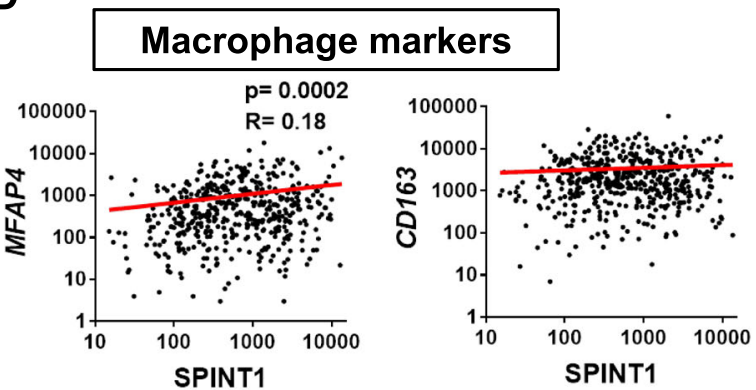

E

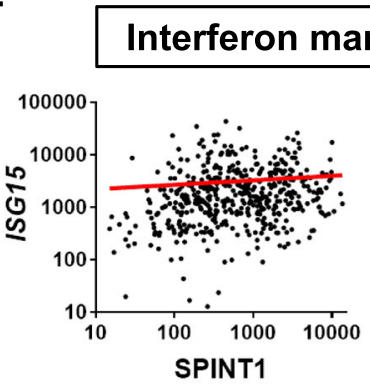

\section{kers}

Fig. 2 (See legend on next page.) 
(See figure on previous page.)

Fig. 2 SPINT1 expression correlates with aggressiveness marker expression in human SKCM biopsies. Correlation of SPINT1 gene expression with those of the melanocyte differentiation markers SOX10, TYR and DCT (a) the EMT markers ZEB1, ZEB2, TWIST1 and TWIST2 (b)), the inflammation markers TNFA, TNFR1, TNFR2, IL8 (CXCL8), CXCR2, IL6 and IL6R (c), the macrophage markers MFAP4 and CD163 (d) and the interferon markers ISG15 and IFIT1 (e) in human SKCM biopsies of the TCGA cohort. The statistical significance of the correlation was determined using Pearson's correlation coefficient. A linear regression-fitting curve in red is also shown

larger than their control counterparts (Fig. 6d). In addition, the recipients injected with Spintla-deficient SKCM cells developed larger tumors spanning the entire dorsal area and even exceed the notochord line and grew vertically, a clear aggressiveness signature of SKCM (Fig. 6d).

To further investigate the aggressiveness potential of Spint1a-deficient SKCMs, a serial dilution assay was performed following the work-flow previously described in
Additional file 1: Figure S1. Cells from both Spint1a-deficient and wild type SKCMs were serially diluted and 3 different numbers of cells (30,000 cells, 100,000 cells and 300,000 cells) were transplanted into recipients as described above. Notably, while 30,000 and 100,000 Spint1a-deficient SKCM cells were able to engraft and the tumor grew over the time, wild type SKCMs hardly grew (Additional file 1: Figure S1A, S1B). However,

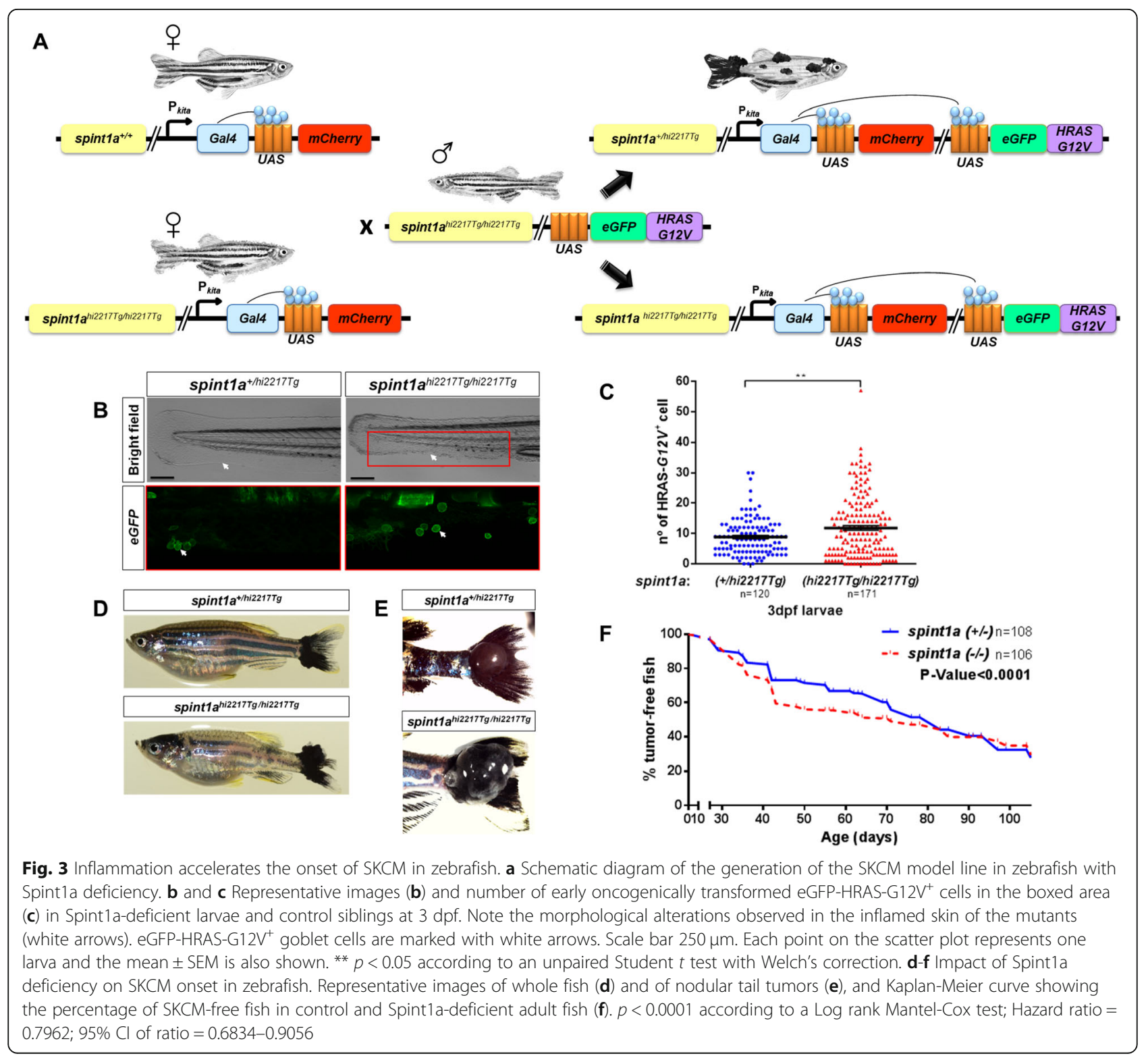


A

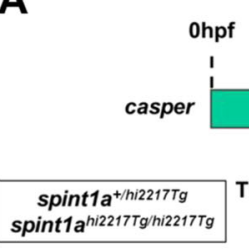

spint1a
spint1 $1 a^{\text {hi2217Tg/hi2217Tg }}$

TUMOR SAMPLING AND

MELANOMA CELL

DISAGGREGATION

$\downarrow$

MELANOMA CELL

STAINING

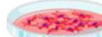

$\downarrow$

MELANOMA CELL

INJECTION IN THE YOLK SAC

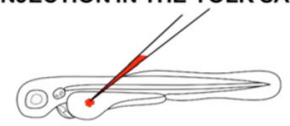

C

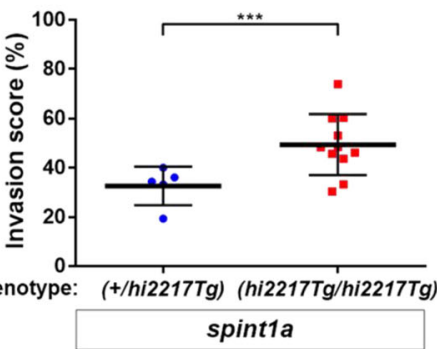

E

Tumor genotype: (+/hi2217Tg) (hi2217Tg/hi2217Tg)

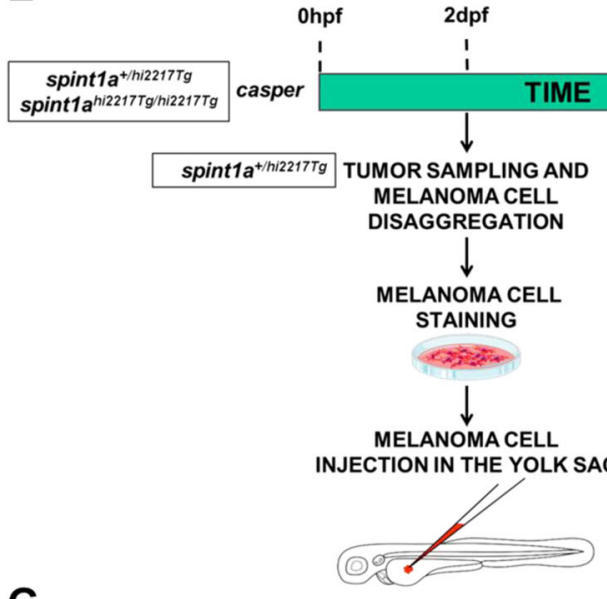

G

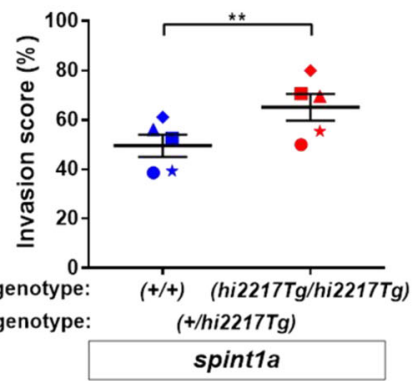

B

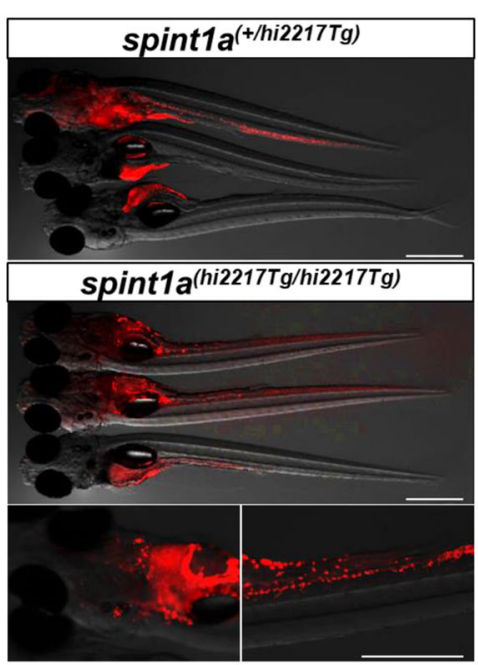

D

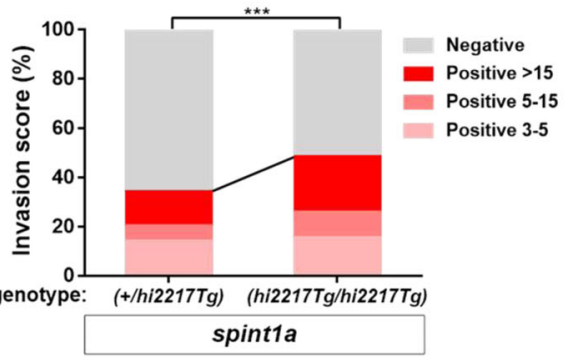

$F$ Genotype

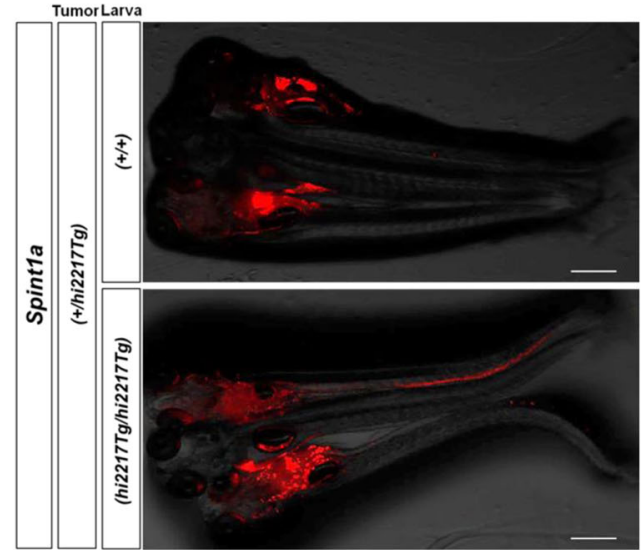

H

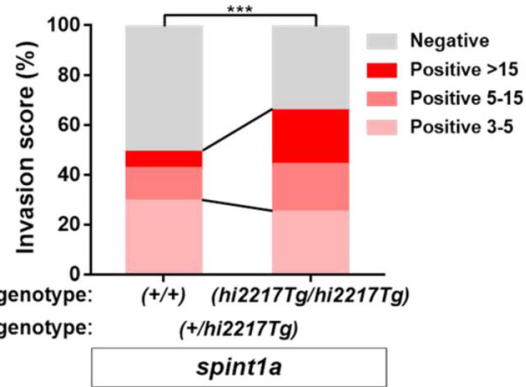

Fig. 4 (See legend on next page.) 
(See figure on previous page.)

Fig. 4 Spint1a deficiency is required at cell autonomous and non-autonomous levels to enhance SKCM cell dissemination in a zebrafish larval allotrasplantation model. Analysis of dissemination of control and Spint1a-deficient SKCM allotransplants in wild type larvae (a-d) and SKCM allotransplants in wild type and Spint1a-deficient larvae (e-h). $\mathbf{a}$ and $\mathbf{e}$ Experimental design. $\mathbf{b}$ and $\mathbf{f}$ Representative images (overlay of bright field and red channels) of SKCM invasion at 5 dpi. Bars: $500 \mu \mathrm{m}$. c and $\mathbf{g}$ Percentage of invaded larvae for both tumor genotypes. Each dot represents a single tumor and the mean \pm SEM is also shown. ${ }^{* *} p<0.01,{ }^{* *} p<0.0001$ according to unpaired Student $t$ test. $\mathbf{d}$ and $\mathbf{h}$ Number of tumor foci per larva. ${ }^{* * *} p<0.0001$ according to Chi-square Tests

injection of 300,000 Spint1a-deficient SKCM cells resulted in large tumor spanning the entire dorsal area and invading part of the ventral cavity (Additional file 1: Figure S1C), confirming previous results. Collectively, all these results confirm that Spint1a deficiency enhances SKCM aggressiveness.

\section{Spint1a deficiency promotes SKCM dedifferentiation and inflammation}

To understand the mechanisms involved in the Spintlamediated aggressiveness of SKCM, the expression of genes encoding important biomarkers was analyzed by RTqPCR. The mRNA levels of sox10, tyr, dct and mitfa were lower in Spint1a-deficient SKCMs than in their wild type counterparts (Additional file 1: Figure S2). In addition, while the transcript levels of $m m p 9$ and slug were similar in Spint1a-deficient and wild type SKCM, $c d h 1$ levels significantly decreased in Spint1a-deficient compared to wild type SKCM (Additional file 1: Figure S2).

We next analyzed genes encoding key inflammatory molecules and immune cell markers, including the proinflammatory cytokine Il1b, the neutrophil markers Lyz

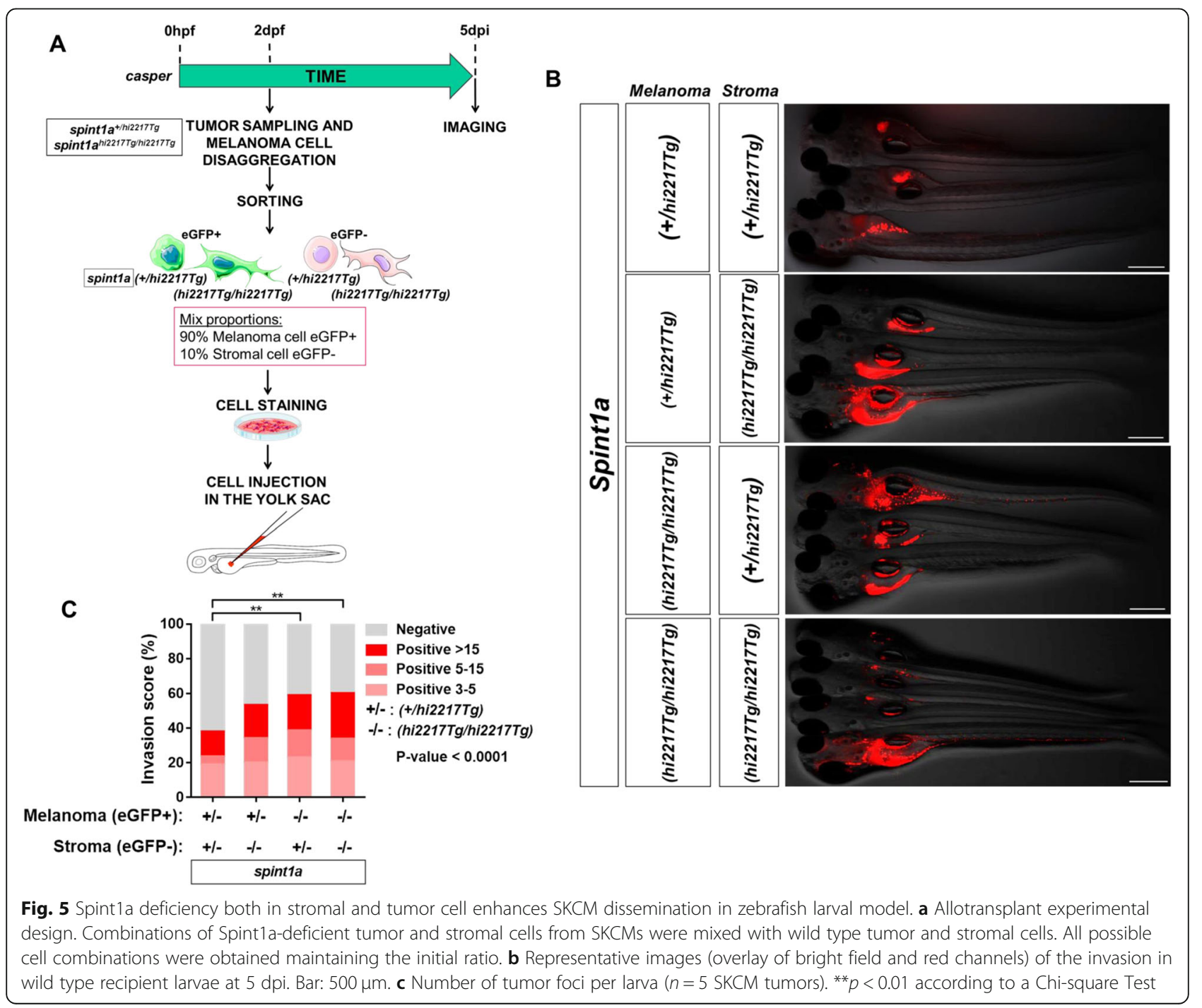




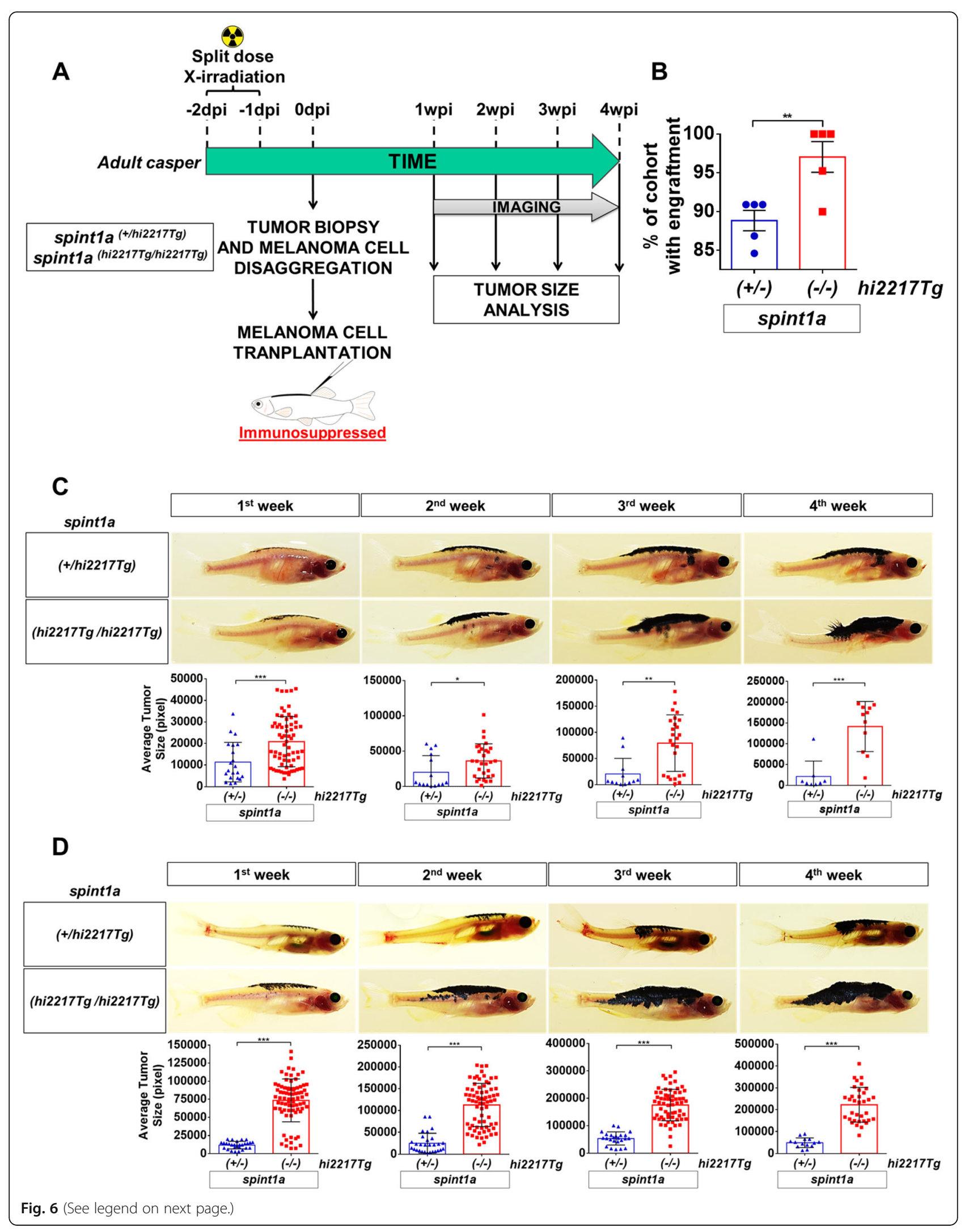


(See figure on previous page.)

Fig. 6 Spint1a-deficient SKCM shows enhanced aggressiveness in adult zebrafish allotransplantation assays. a Experimental workflow of adult allotransplantation experiments in pre-irradiated adult casper zebrafish. b Percentage of engraftment for both control and Spint1a-deficient tumors. Each dot represents a single SKCM tumor and the mean \pm SEM is also shown $(n=5)$. $\mathbf{c}$ and $\mathbf{d}$ Representative images and average tumor size (pixels) from 1 to 4 weeks post-transplant of primary (c) and secondary (d) transplants. Each dot corresponds to a recipient-transplanted fish and the mean \pm SEM is also shown. $\mathbf{b}-\mathbf{d}{ }^{*} p<0.05,{ }^{* *} p<0.01,{ }^{* *} p<0.001$ according to unpaired Student $t$ test

and Mpx, the macrophage marker Mpeg1, and the ISGs B2m, Mxb and Pkz, in Spint1a-deficient and wild type SKCMs (Additional file 1: Figure S2). Curiously, it was found that while $i l 1 b, l y z$ and $m p x$ mRNA levels were not affected by Spintla deficiency, those of mpeg1 were elevated in Spint1a-deficient SKCMs. Furthermore, the ISGs $b 2 m, m x b$ and $p k z$ genes showed enhanced mRNA levels in Spintla-deficient SKCMs. These results point out to altered immune surveillance and tumor cell dedifferentiation promoted by Spintla-deficiency in SKCM.

\section{Discussion}

The relationships between inflammation and cancer are ambiguous. Although it is estimated that underlying infections and inflammatory responses are linked to $15-20 \%$ of all deaths from cancer worldwide [11], immunosuppression is known to increase the risk for certain tumors [10]. Furthermore, immunotherapy is considered the most promising cancer therapy for the next future [34]. In this study, we have developed a preclinical zebrafish model to study the role of SPINT1-driven skin chronic inflammation in melanoma. We found that Spintla deficiency is required at both cell autonomous and non-autonomous levels to enhance cell dissemination of SKCM by promoting tumor dedifferentiation and altered immune surveillance. These results may have important clinical impact, since genetic alterations of SPINT1 were found in $10 \%$ of SKCM patients and correlated with altered cell cycle, differentiation and innate and adaptive immune signaling pathways and, more importantly, with a poor prognosis. In addition, a positive correlation of both inflammation and macrophage marker with SPINT1 levels was found in tumor samples, fitting the increased number of TAM in SKCM with high SPINT1 levels. However, a negative correlation of SPINT1 and EMT marker levels was observed, suggesting that SPINT1 levels critically regulate tumor and immune cell crosstalk. Therefore, both high and low levels of SPINT1 result in an unbalanced crosstalk between tumor cells and their microenvironment promoting higher SKCM aggressiveness.

Another interesting observation is that SPINT1 transcript levels positively correlated with macrophage infiltration, but not neutrophil one, in SKCM tumor samples. Similarly, our gene expression studies also suggest that Spint1a regulates macrophage infiltration in the zebrafish SKCM model. Curiously, activated neutrophils in a condition of repeated wounding have been shown to interact with pre-neoplastic cells promoting their proliferation through the release of prostaglandin E2 and, more importantly, SKCM ulceration correlates with increased neutrophil infiltration and tumor cell proliferation, which are both associated with poor prognosis [35]. Although we found a robust positive correlation between the transcript levels of SPINT1 and CXCR2, which encodes a major IL-8 receptor involved in SKCM neutrophil infiltration [36], CXCR2 has also been shown to promote tumor-induced angiogenesis and increased proliferation [37-39] and, therefore, the SPINT1/CXCR2 axis may regulate SKCM aggressiveness by neutrophil-independent pathways.

The zebrafish model developed in this study uncovered a role for Spintla in facilitating oncogenic transformation which probably accelerates the SKCM onset. Curiously, Spintla deficiency seems to have a prominent role in oncogenic transformation accelerating SKCM onset in vivo. However, the strong oncogenic activity of HRAS-G12V, which is even able to induce melanoma without the need of coadjuvating mutations in tumor suppressors, results in a similar tumor burden in wild type and Spint1a-deficient fish at later stages. It would be interesting, therefore, to analyze in future studies the impact of Spintla in SKCM models driven by other major oncogenes, such as Braf, Kras, Nras and Nf1. Although SPINT1 is a serine protease inhibitor with several targets, including ST14 and HGFA, deregulation of the SPINT1/ST14 axis leads to spontaneous squamous cell carcinoma in mice [17] and keratinocyte hyperproliferation in zebrafish $[19,20]$ preceded by skin inflammation in both models. In addition, intestine-specific Spint1 deletion in mice induces the activation of the master inflammation transcription factor NF- $\mathrm{kB}$ and accelerated intestinal tumor formation [40]. Strikingly, pharmacological inhibition of NF-KB activation reduces the formation of intestinal tumors in Spint1-deficient ApcMin/+ mice [40], unequivocally demonstrating that Spint1-driven inflammation promotes tumorigenesis.

The SKCM allotransplant assays in larvae revealed for the first time that Spint1a deficiency in both tumor and stromal cells increases SKCM invasiveness. In addition, Spintla deficiency in both cell types does not show enhanced invasiveness compared to Spintla deficiency in either cell type. This is an interesting observation, since SPINT1 is a membrane-bound protein that may, therefore, inhibit their targets in both tumor cell autonomous and non-autonomous manners. However, wild 
type Spintla tumor microenvironment fails to compensate its loss in tumor cells, since Spint1a-deficient tumor cells show enhanced invasiveness in wild type larvae and adult recipients, and vice versa. Importantly, transplantation experiments of serial diluted SKCM cells revealed the crucial cell-autonomous role of Spintla in inhibiting tumor aggressiveness. Similarly, loss of SPINT1 in human pancreatic cancer cells promotes ST14-dependent metastasis in nude mouse orthotopic xenograft models [41]. We observed that genetic alterations in SPINT1 transcript levels in SKCM patient samples negatively correlated with EMT markers and that Spint1a-deficient zebrafish SKCM showed reduced $c d h 1$ mRNA levels. EMT phenotype switching has been shown to be involved in acquisition of metastatic properties in the vertical growth phase of SKCM [42] and loss of E-cadherin, with gain of $\mathrm{N}$-cadherin and osteonectin, was associated with SKCM metastasis [43]. Importantly, the presence of aberrant E-cadherin expression in primary and metastatic SKCM is associated with a poor overall patient survival [44]. Therefore, our results suggest that SPINT1 loss may facilitate metastatic invasion of human SKCM through EMT phenotype switching.

\section{Conclusions}

We have developed a preclinical model to study the role of altered expression of SPINT1 in early transformation, progression and metastatic invasion in SKCM. This model has revealed that Spintla deficiency facilitates oncogenic transformation, regulates the tumor/immune microenvironment crosstalk and is associated to SKCM aggressiveness. In addition, Spint1a deficiency in either tumor or microenvironment compartment increases SKCM aggressiveness. The high prevalence of SPINT1 genetic alterations in SKCM patients and their association with a poor prognosis, point out to SPINT1 as a novel therapeutic target for the treatment of SKCM.

\section{Additional file}

Additional file 1: Figure S1. Spint1a-deficient SKCM shows enhanced aggressiveness in adult zebrafish allotransplantation assays. Control and Spint1a deficient SKCMs were disaggregated and 30,000 (a), 100,000 (b) and 300,000 cells (c) were injected subcutaneously in pre-irradiated adult casper zebrafish. Fish were analyzed for average tumor size (pixels) from 1 to 4 weeks post-transplant. Representative images and quantification of the average tumor size are shown. Each dot corresponds to a recipienttransplanted fish and the mean \pm SEM is also shown ( $n=2$ SKCM tumors). ${ }^{*} p<0.05,{ }^{* * *} p<0.001$ according to unpaired Student $t$ test. Figure S2. Expression analysis of differentiation melanocyte, EMT, inflammation and immune cell markers in zebrafish SKCM. The mRNA levels of the genes encoding the differentiation melanocyte markers sox10, mitfa, tyr and $d c t$, the EMT markers $c d h$ 1, slug and $m m p 9$, the inflammation marker $i / 1 b$, the neutrophil markers lyz and $m p x$, the macrophage marker mpeg 1, and the ISGs $b 2 m, m \times b$ and pkz were analyzed by RT-qPCR in control and Spint1a-deficient SKCMs. ${ }^{*} p<0.05,{ }^{* *} p<0.01$ according to one-tailed Student $t$ test. Table S1. Primers used in this study for RT-qPCR. The gene symbols followed the Zebrafish Nomenclature Guidelines (http:// zfin.org/zf_info/nomen.html/). ENA, European Nucleotide Archive (http:// www.ebi.ac.uk/ena/). (PDF 496 kb)

\section{Abbreviations}

ANOVA: Analyzed by analysis of variance; dpf: Days post-fertilization; Dil: 1,1'di-octa-decyl-3,3,3',3'-tetra-methyl-indo-carbo-cya-nine perchlorate; eGFP: Enhanced green fluorescent protein; FBS: Fetal bovine serum; HAl1: Hepatocyte growth factor activator inhibitor 1; HGFA: Hepatocyte growth factor activator; hpf: Hours post-fertilization; SKCM: Skin cutaneous melanoma; Spint1: Serine protease inhibitor, kunitz-type, 1; ST14: Suppression of tumorigenicity 14; TCGA: The Cancer Genome Atlas; TAM: Tumorassociated macrophages; TAN: Tumor-associated neutrophils

\section{Acknowledgments}

We strongly acknowledge P. Martínez for his excellent technical assistance with zebrafish maintenance.

\section{Authors' contributions}

VM conceived the study; EGA, LIZ, CG and VM designed research; EGA, SIM, DGM, IF and CG performed research; EGA, SIM, DGM, IF, LIZ, MCM, MLC, CG and VM analyzed data; and EGA, CG and VM wrote the manuscript with minor contribution from other authors. All authors read and approved the final manuscript.

\section{Funding}

This work was supported by the Spanish Ministry of Science, Innovation and Universities (grants BIO2014-52655-R and BIO2017-84702-R to VM and PI13/ 0234 to MLC), all co-funded with Fondos Europeos de Desarrollo Regional/ European Regional Development Funds), Fundación Séneca-Murcia (grant 19400/PI/14 to MLC), the University of Murcia (postdoctoral contracts to DGM), and the European Union Seventh Framework Programme-Marie Curie COFUND (FP7/2007-2013) under UMU Incoming Mobility Programme ACTion (U-IMPACT) Grant Agreement 267143. The funders had no role in study design, data collection and analysis, decision to publish, or preparation of the manuscript.

\section{Availability of data and materials}

Further information and requests for resources and reagents should be directed to and will be fulfilled by the Lead Contact, Victoriano Mulero (vmulero@um.es).

\section{Ethics approval and consent to participate}

The experiments complied with the Guidelines of the European Union Council (Directive 2010/63/EU) and the Spanish RD 53/2013. Experiments and procedures were performed as approved by the Consejería de Agua, Agricultura, Ganadería y Pesca de la CARM (authorization number \# A13180602)

\section{Consent for publication}

Not applicable.

\section{Competing interests}

L.I.Z. is a founder and stockholder of Fate Therapeutics, Inc., Scholar Rock and Camp4 Therapeutics.

\section{Author details}

${ }^{1}$ Departamento de Biología Celular e Histología, Facultad de Biología, Universidad de Murcia, Murcia, Spain. ${ }^{2}$ Instituto Murciano de Investigación Biosanitaria (IMIB)-Arrixaca, Murcia, Spain. ${ }^{3}$ Department of Stem Cell and Regenerative Biology, Harvard Stem Cell Institute, Cambridge, MA, USA. ${ }^{4}$ Stem Cell Program and Division of Hematology/Oncology, Boston Children's Hospital and Dana-Farber Cancer Institute, Howard Hughes Medical Institute, Harvard Stem Cell Institute, Harvard Medical School, Boston, MA, USA.

${ }^{5}$ Laboratory of Experimental Cancer Biology, Cibio, University of Trento, Trento, Italy. ${ }^{6}$ Hospital Clínico Universitario Virgen de la Arrixaca, IMIB-Arrixaca, Murcia, Spain. ${ }^{7}$ Present Address: Unit of Cell and Developmental Biology, Department of Biology, University of Pisa, S.S. 12 Abetone e Brennero 4, Pisa, Italy. 
Received: 30 July 2019 Accepted: 23 August 2019

\section{Published online: 13 September 2019}

\section{References}

1. Wellbrock C, Arozarena I. The complexity of the ERK/MAP-kinase pathway and the treatment of melanoma skin cancer. Front Cell Dev Biol. 2016;4:33.

2. Schadendorf D, Hauschild A. Melanoma in 2013: melanoma--the run of success continues. Nat Rev Clin Oncol. 2014;11(2):75-6.

3. van Rooijen E, Fazio M, Zon LI. From fish bowl to bedside: the power of zebrafish to unravel melanoma pathogenesis and discover new therapeutics. Pigment Cell Melanoma Res. 2017;30(4):402-12.

4. NIH. Cancer stat facts: melanoma of the skin: National Cancer Institute; 2019 https://seer.cancer.gov/statfacts/html/melan.htm

5. Schadendorf D, Fisher DE, Garbe C, Gershenwald JE, Grob JJ, Halpern A, et al. Melanoma. Nat Rev Dis Primers. 2015;1:15003.

6. Hoerter JD, Bradley P, Casillas A, Chambers D, Weiswasser B, Clements $L$, et al. Does melanoma begin in a melanocyte stem cell? J Skin Cancer. 2012;2012:571087

7. Kaufman CK, Mosimann C, Fan ZP, Yang S, Thomas AJ, Ablain J, et al. A zebrafish melanoma model reveals emergence of neural crest identity during melanoma initiation. Science. 2016;351(6272):aad2197.

8. Santoriello C, Gennaro E, Anelli V, Distel M, Kelly A, Koster RW, et al. Kita driven expression of oncogenic HRAS leads to early onset and highly penetrant melanoma in zebrafish. PLoS One. 2010;5(12):e15170.

9. Nguyen N, Couts KL, Luo Y, Fujita M. Understanding melanoma stem cells. Melanoma Manag. 2015;2(2):179-88.

10. Shalapour S, Karin M. Immunity, inflammation, and cancer: an eternal fight between good and evil. J Clin Invest. 2015;125(9):3347-55.

11. Maru GB, Gandhi K, Ramchandani A, Kumar G. The role of inflammation in skin cancer. Adv Exp Med Biol. 2014;816:437-69.

12. Tang L, Wang K. Chronic inflammation in skin malignancies. J Mol Signal. 2016;11:2.

13. Lin CY, Anders J, Johnson M, Sang QA, Dickson RB. Molecular cloning of cDNA for matriptase, a matrix-degrading serine protease with trypsin-like activity. J Biol Chem. 1999;274(26):18231-6.

14. Benaud C, Dickson RB, Lin CY. Regulation of the activity of matriptase on epithelial cell surfaces by a blood-derived factor. Eur J Biochem. 2001;268(5):1439-47.

15. Tseng IC, Chou FP, Su SF, Oberst M, Madayiputhiya N, Lee MS, et al. Purification from human milk of matriptase complexes with secreted serpins: mechanism for inhibition of matriptase other than HAI-1. Am J Physiol Cell Physiol. 2008;295(2):C423-31.

16. Shimomura T, Denda K, Kitamura A, Kawaguchi T, Kito M, Kondo J, et al. Hepatocyte growth factor activator inhibitor, a novel Kunitz-type serine protease inhibitor. J Biol Chem. 1997;272(10):6370-6.

17. List K, Szabo R, Molinolo A, Sriuranpong V, Redeye V, Murdock T, et al. Deregulated matriptase causes ras-independent multistage carcinogenesis and promotes ras-mediated malignant transformation. Genes Dev. 2005; 19(16):1934-50.

18. List K. Matriptase: a culprit in cancer? Future Oncol. 2009;5(1):97-104.

19. Mathias JR, Dodd ME, Walters KB, Rhodes J, Kanki JP, Look AT, et al. Live imaging of chronic inflammation caused by mutation of zebrafish Hai1. J Cell Sci. 2007;120(Pt 19):3372-83.

20. Carney TJ, von der Hardt S, Sonntag C, Amsterdam A, Topczewski J, Hopkins $\mathrm{N}$, et al. Inactivation of serine protease Matriptase1a by its inhibitor Hai1 is required for epithelial integrity of the zebrafish epidermis. Development. 2007;134(19):3461-71.

21. Westerfield M. The zebrafish book. A guide for the laboratory use of zebrafish Danio* (Brachydanio) rerio. Eugene: University of Oregon Press; 2000

22. White RM, Sessa A, Burke C, Bowman T, LeBlanc J, Ceol C, et al. Transparent adult zebrafish as a tool for in vivo transplantation analysis. Cell Stem Cell. 2008;2(2):183-9.

23. Dang M, Henderson RE, Garraway LA, Zon LI. Long-term drug administration in the adult zebrafish using oral gavage for cancer preclinical studies. Dis Model Mech. 2016;9(7):811-20.

24. Marques IJ, Weiss FU, Vlecken DH, Nitsche C, Bakkers J, Lagendijk AK, et al. Metastatic behaviour of primary human tumours in a zebrafish xenotransplantation model. BMC Cancer. 2009;9:128.

25. Pfaffl MW. A new mathematical model for relative quantification in real-time RT-PCR. Nucleic Acids Res. 2001;29(9):e45.
26. Coussens LM, Werb Z. Inflammation and cancer. Nature. 2002; 420(6917):860-7.

27. Colegio OR, Chu NQ, Szabo AL, Chu T, Rhebergen AM, Jairam V, et al. Functional polarization of tumour-associated macrophages by tumourderived lactic acid. Nature. 2014;513(7519):559-63.

28. Gordon SR, Maute RL, Dulken BW, Hutter G, George BM, McCracken MN, et al. PD-1 expression by tumour-associated macrophages inhibits phagocytosis and tumour immunity. Nature. 2017:545(7655):495-9.

29. Zaidi MR, Davis S, Noonan FP, Graff-Cherry C, Hawley TS, Walker RL, et al. Interferon-gamma links ultraviolet radiation to melanomagenesis in mice. Nature. 2011:469(7331):548-53.

30. Ronnstrand L, Phung B. Enhanced SOX10 and KIT expression in cutaneous melanoma. Med Oncol. 2013;30(3):648.

31. Ordonez NG. Value of melanocytic-associated immunohistochemical markers in the diagnosis of malignant melanoma: a review and update. Hum Pathol. 2014:45(2):191-205.

32. Wang $H$, Yang L, Wang D, Zhang Q, Zhang L. Pro-tumor activities of macrophages in the progression of melanoma. Hum Vaccin Immunother. 2017;13(7):1556-62.

33. Feng $Y$, Santoriello C, Mione M, Hurlstone A, Martin P. Live imaging of innate immune cell sensing of transformed cells in zebrafish larvae: parallels between tumor initiation and wound inflammation. PLoS Biol. 2010;8(12):e1000562.

34. Carreau N, Pavlick A. Revolutionizing treatment of advanced melanoma with immunotherapy. Surg Oncol. 2019. https://doi.org/10.1016/j.suronc.2019.01.002.

35. Antonio N, Bonnelykke-Behrndtz ML, Ward LC, Collin J, Christensen IJ, Steiniche T, et al. The wound inflammatory response exacerbates growth of pre-neoplastic cells and progression to cancer. EMBO J. 2015;34(17):2219-36.

36. Jablonska J, Wu CF, Andzinski L, Leschner S, Weiss S. CXCR2-mediated tumor-associated neutrophil recruitment is regulated by IFN-beta. Int J Cancer. 2014;134(6):1346-58.

37. Singh S, Sadanandam A, Nannuru KC, Varney ML, Mayer-Ezell R, Bond R, et al. Small-molecule antagonists for CXCR2 and CXCR1 inhibit human melanoma growth by decreasing tumor cell proliferation, survival, and angiogenesis. Clin Cancer Res. 2009;15(7):2380-6.

38. Gabellini C, Gomez-Abenza E, Ibanez-Molero S, Tupone MG, Perez-Oliva AB, de Oliveira S, et al. Interleukin 8 mediates bcl-xL-induced enhancement of human melanoma cell dissemination and angiogenesis in a zebrafish xenograft model. Int J Cancer. 2018;142(3):584-96.

39. Gabellini C, Trisciuoglio D, Desideri M, Candiloro A, Ragazzoni Y, Orlandi A, et al. Functional activity of CXCL8 receptors, CXCR1 and CXCR2, on human malignant melanoma progression. Eur J Cancer. 2009;45(14):2618-27.

40. Kawaguchi M, Yamamoto K, Kanemaru A, Tanaka H, Umezawa K, Fukushima $\mathrm{T}$, et al. Inhibition of nuclear factor-kappaB signaling suppresses Spint1deletion-induced tumor susceptibility in the ApcMin/+ model. Oncotarget. 2016;7(42):68614-22.

41. Ye J, Kawaguchi M, Haruyama Y, Kanemaru A, Fukushima T, Yamamoto K, et al. Loss of hepatocyte growth factor activator inhibitor type 1 participates in metastatic spreading of human pancreatic cancer cells in a mouse orthotopic transplantation model. Cancer Sci. 2014;105(1):44-51.

42. Bennett DC. How to make a melanoma: what do we know of the primary clonal events? Pigment Cell Melanoma Res. 2008;21(1):27-38.

43. Alonso SR, Tracey L, Ortiz P, Perez-Gomez B, Palacios J, Pollan M, et al. A high-throughput study in melanoma identifies epithelial-mesenchymal transition as a major determinant of metastasis. Cancer Res. 2007;67(7): 3450-60.

44. Yan S, Holderness BM, Li Z, Seidel GD, Gui J, Fisher JL, et al. Epithelialmesenchymal expression phenotype of primary melanoma and matched metastases and relationship with overall survival. Anticancer Res. 2016; 36(12):6449-56

\section{Publisher's Note}

Springer Nature remains neutral with regard to jurisdictional claims in published maps and institutional affiliations. 